\title{
APPROXIMATION BY LINEAR COMBINATIONS OF TRANSLATES OF A SINGLE FUNCTION
}

\author{
DiNH DŨNG AND VU NHAT HUY*
}

\begin{abstract}
We study approximation of periodic functions by arbitrary linear combinations of $n$ translates of a single function. We construct some linear methods of this approximation for univariate functions in the class induced by the convolution with a single function, and prove upper bounds of the $L^{p}$-approximation convergence rate by these methods, when $n \rightarrow \infty$, for $1 \leqslant p \leqslant \infty$. We also generalize these results to classes of multivariate functions defined as the convolution with the tensor product of a single function. In the case $p=2$, for this class, we also prove a lower bound of the quantity characterizing best approximation of by arbitrary linear combinations of $n$ translates of arbitrary function.
\end{abstract}

Mathematics subject classification (2020): 41A46, 41A63, 42A99.

Keywords and phrases: Function spaces induced by the convolution with a given function; Approximation by arbitrary linear combinations of $n$ translates of a single function.

\section{REFERENCES}

[1] D. DŨNG AND C. A. Michelli, Multivariate approximation by translates of the Korobov function on Smolyak grids, Journal of Complexity, 29 (2013), 424-437.

[2] D. DŨNg AND C. A. Micchelli, Corrigendum to "Multivariate approximation by translates of the Korobov function on Smolyak grids", [J. Complexity 29 (2013), 424-437], J. Complexity 35 (2016), 124-125.

[3] D. Dũng, C. A. Micchelli And V. N. HuY, Approximation by translates of a single function of functions in space induced by the convolution with a given function, Applied Mathematics and Computation 361 (2019), 777-787.

[4] E. Landau, Ungleichungen für zweimal differenzierbare Funktionen, Proc. London Math. Soc. 13 (1913), 43-49.

[5] V. MAIOROv, Almost optimal estimates for best approximation by translates on a torus, Constructive Approx. 21 (2005), 1-20.

[6] M. MagGioni And H. N. Mhaskar, Diffusion polynomial frames on metric measure spaces, Appl. Comput. Harmon. Anal. 24 (2008), 329-353.

[7] H. N. Mhaskar, Eignets for function approximation on manifolds, Appl. Comput. Harmon. Anal. 29 (2010), 63-87. 